\title{
Cloning and functional prediction of differentially expressed genes in the leaves of Glycine max parents and hybrids at the seedling stage
}

\author{
J. Zhang, D. Yao, P. Wang, S.Y. Guan, J. Ma and Y.P. Fu \\ College of Agronomy of Jilin Agricultural University, Changchun, Jilin, China \\ Corresponding author: P. Wang \\ E-mail: zhangjun9696@sina.cn
}

Genet. Mol. Res. 13 (3): 5474-5483 (2014)

Received May 3, 2013

Accepted September 9, 2013

Published February 13, 2014

DOI http://dx.doi.org/10.4238/2014.February.13.15

\begin{abstract}
Here, we compare the molecular mechanism of soybean heterosis through the differential expression of basic cloning. Specifically, we cloned 22 differentially expressed cDNA fragments from hybrid combinations of Jilin 38 x EXP (which had obvious yield advantages) and their parents. In addition, we compared the homology of these fragments and predicted their functions. Cloning differentially expressed genes included the identification of the calmodulin binding protein, $18 \mathrm{~S}$ ribosomal gene, $26 \mathrm{~S}$ ribosomal gene, soybean satellite DNA, soybean acid phosphatase, soybean chlorophyll a/b-binding protein II (Cab-6) gene, soybean chloroplast PI 437654 gene, soybean PPR protein gene, and other fragments with unknown functions. In conclusion, the cloning and functional prediction of differentially expressed soybean genes in this study is anticipated to provide valuable information for studies on the molecular mechanism of heterosis.
\end{abstract}

Key words: Glycine max; Soybean; Gene cloning; Heterosis; DDRT 


\section{INTRODUCTION}

Heterosis has been widely used to enhance crops around the world. For example, maize heterosis led to the popularization of a single cross hybrid in the United States during the early 1960s. Another maize hybrid strain was also popularized in China. Rice heterosis led to a hybrid rice combination first being bred in China in 1975, which was widely promoted in 1976. Soybean exhibits stronger heterosis; however, breakthroughs have been made in recent years for its utilization. Zhao et al. (2004) at the Jilin Academy of Agricultural Sciences bred the first hybrid soybean variety "HybSoy 1", which was cultivated with the cultivar soybean 167 as the cytoplasm, leading to $20 \%$ increase in yield compared to Jilin 30 .

The utilization of crop plant heterosis has generated great benefits in production; however, research about the mechanism of heterosis remains limited, particularly at the molecular level. Studies about the genetic mechanism of heterosis have focused on the relationship between genetic differences and heterosis, and have not promoted the heterosis mechanism of crops. The quantitative trait loci (QTL) positioning method was developed in the late 1980s, and laid the foundation for investigation of the relationship between QTL heterozygosity, its interaction forms, and heterosis formation. Stuber (1995), Xiao et al. (1995), and Li et al. (2000) further advanced our understanding about the mechanism of heterosis; however, the molecular mechanism of heterosis formation has yet to be elucidated. Cheng et al. (1997), Xiong (1999), and Ni et al. (2000) conducted preliminarily studies on maize, rice, and wheat, respectively, with respect to gene expression, by the technology of differential display of mRNA reverse transcription polymerase chain reaction (DDRT-PCR).

With the development and application of soybean heterosis, it becomes necessary to research the molecular mechanism of soybean heterosis. Cerna et al. (1997) studied the hybridization of heterosis with respect to soybean seed yield and the RFLP molecular marker. The authors concluded that RFLP labeled diversity is not related to the heterosis of seed yield. In addition, genetic distance was only slightly related to seed yield. Therefore, the authors concluded that the RFLP marker serves as a poor predictor of heterosis. Zhang et al. (2003) investigated the possibility of predicting the heterosis of 22 soybeans in an $\mathrm{F}_{1}$ generation hybridized through 10 bred soybean varieties from 6 provinces and cities, based on the molecular genetic distance between parents using SSR markers. The authors concluded that there is a quadratic curved relationship between the genetic distance and above-mid-parent heterosis and high parent heterosis for the 100 kernel weight in a small proportion of the whole $\mathrm{F}_{1}$ generation that had a partially similar genetic background; hence, SSR markers could not satisfactorily predict soybean heterosis.

Similar studies on the mechanism of soybean heterosis using DDRT-PCR technology have not been reported in China. The differential display technique is used in other countries around the world to separate the LEA5-like cDNA protein (late embryogenesis abundant, LEA) (Burns et al., 1996) and induce soybean to synthesize the cDNA of cytochrome P450S (P450dependent enzymes, P450s) (Schopfer and Ebel, 1998). In addition, Vladimir (2001) investigated the differential expression of aluminum tolerant and aluminum intolerant soybean strain genes by the DDRT-PCR technique under the selection pressure of aluminum.

DDRT-PCR technology was first established by Liang and Pardee (1992). Compared to SSR and RFLP molecular marker technology, this technology quickly and effectively identifies and clones differentially expressed genes between 2 or more parallel materials. This technique has several benefits, including the use of smaller amounts of DNA, high sensitivity, 
simple operation, rapid, and the ability to analyze low-abundance mRNA. As a result, this method is widely used in studies of plant gene isolation, cloning, and expression.

We studied the relationship between differential expression patterns of the soybean leaf gene at the seedling stage, and identified the heterosis of various agronomic and quality properties by DDRT-PCR technology (Zhang et al., 2010). We found that some differential expression patterns are significantly or extremely significantly correlated with agronomic and quality properties; however, genes change expression that may be used to further understand the molecular mechanism of heterosis. Therefore, the cloning of differentially expressed genes is expected to further facilitate our understanding about the relationship of these genes and the heterosis of various trait properties. At present, several researchers have cloned some genes in maize, rice, and wheat (Cheng et al., 1997); however, reports on the cloning of differentially expressed genes of soybean at the seedling stage have not been published. Therefore, it is necessary to conduct the cloning and sequencing of differentially expressed genes of soybean at the seedling stage. This experiment recovered and cloned expressed cDNA fragments (verified by reverse Northern hybridization method) of the hybrid combination Jilin No. 38xEXP, which has obvious heterosis of soybean yield characteristics (Wang et al., 2008), using the differential display technique of mRNA. These fragments were sequenced and their functions were predicted.

\section{MATERIAL AND METHODS}

\section{Materials}

The hybrid combination Jilin No. 38xEXP, which has strong heterosis of soybean yield characteristics, and the leaves of its parents at the seedling stage were used as the test materials, and were stored in a refrigerator at $-80^{\circ} \mathrm{C}$.

\section{RT-PCR and differential display}

The soybean leaf RNA was extracted using the plant RNA extraction kit (Beijing Applygen Technologies Inc., Beijing, China) following manufacturer protocols. Subsequently, ultraviolet absorption detection and gel detection of the total RNA samples was conducted, following manufacturer protocols in the Qligotex Manual (Qiagen Company, Shanghai, China).

Primers were designed according to the published literature ( $\mathrm{Wu}, 2004)$, and were synthesized by the Beijing SunBio Company (Beijing, China). Anchored primers at the 3'-end included: R1: 5'-AAGCTTTTTTTTTTTA-3'; R2: 5'-AAGCTTTTTTTTTTTC-3'; and R3: 5'-AAGCTTTTTTTTTTTG-3'. Oligonucleotide primers at the 5'-end included: L1: 5'-TGCCGAAGCTTTGGTAGC-3'; L2: 5'-TGCCGAAGCTTTGGTCAG-3'; L3: 5'-TGCCGAAGCTTGATTCCG-3'; L4: 5'-TGCCGAAGCTTTGGTGAC-3'; and L5: 5'-TGCCGAAGCTTTGGTGTC-3'. The 3 anchored primers were matched with the 5 oligonucleotide primers, to form 15 pairs of primers.

The first cDNA chain was synthesized through the reverse transcription of total RNA using the total RNA of the soybean leaves as the template, following manufacturer protocols for the SuperscriptTMII Kit (GIBCO BRL, Gaithersburg, MD, USA). Then PCR amplification was conducted using the first cDNA chain as the template, and using the corresponding anchored primers and random primers. The total volume of the PCR system was $25 \mu \mathrm{L}$, and 
contained $2.5 \mu \mathrm{L}$ reverse transcription product, $2.5 \mu \mathrm{L} 10 \mathrm{X}$ buffer, $2 \mu \mathrm{L}$ dNTP, $2.5 \mu \mathrm{L} \mathrm{MgCl}_{2}$, $0.5 \mu \mathrm{L}$ anchored primers at the 3 '-end, and $0.5 \mu \mathrm{L}$ oligonucleotide primers at the 5 '-end, 0.2 $\mu \mathrm{L}$ Taq DNA polymerase, and $14.3 \mu \mathrm{L}$ ddH2O. PCR conditions were: 40 cycles at $94^{\circ} \mathrm{C}$ for 5 $\min$; $94^{\circ} \mathrm{C}$ for $1 \mathrm{~min}, 40^{\circ} \mathrm{C}$ for $4 \mathrm{~min}, 72^{\circ} \mathrm{C}$ for $1 \mathrm{~min} ; 94^{\circ} \mathrm{C}$ for $45 \mathrm{~s}, 60^{\circ} \mathrm{C}$ for $2 \mathrm{~min}, 72^{\circ} \mathrm{C}$ for $1 \mathrm{~min}$; with a final extension at $72^{\circ} \mathrm{C}$ for $5 \mathrm{~min}$. PCR products were detected by electrophoresis on $4 \%$ denaturing polyacrylamide gel, followed by silver staining.

\section{Recycling and re-amplification of differentially expressed genes}

The boiling method was used for recovery. Specifically, the differentially expressed genes were removed with a scalpel, placed in centrifuge tubes, and then $30 \mu \mathrm{L}$ ultra-pure water was added. The gel block was crushed, boiled in a water bath at $100^{\circ} \mathrm{C}$ for $10 \mathrm{~min}$, kept on ice for $2 \mathrm{~min}$, centrifuged, and then stored at $-20^{\circ} \mathrm{C}$.

For secondary amplification, $2.5 \mu \mathrm{L}$ recovered product was used as the reamplification template. The other reagents were exactly the same as those used in RT-PCR. The PCR amplification procedures were: $94^{\circ} \mathrm{C}$ for $5 \mathrm{~min}$, then 40 cycles at $94^{\circ} \mathrm{C}$ for $45 \mathrm{~s}, 60^{\circ} \mathrm{C}$ for $2 \mathrm{~min}, 72^{\circ} \mathrm{C}$ for $1 \mathrm{~min}$, and finally $72^{\circ} \mathrm{C}$ for $5 \mathrm{~min}$. The secondary amplification products were detected on $1 \%$ agarose gel. The band on the gel was cut off, and then recovered by using the DNA recovery kit.

\section{Verification, cloning, sequencing, and functional analysis of differentially ex- pressed genes}

The probe was labeled using the random prime method by the DIG High Prime DNA Labeling and Detection Starter Kit I No.1745832 (Roche Company, Basel, Switzerland). The cDNA fragment was verified using the reverse Northern hybridization method. Each recovered fragment was simultaneously dotted on 3 membranes. In total, 120 different recovered segments were successively dotted on each hybrid wax, and 3 replicate membranes were hybridized with the total cDNA from the female parent, the hybrid strain, and the male parent, respectively.

The PCR amplification product of the differentially expressed genes (verified by reverse Northern hybridization) was transformed by connecting it to the PMD18-T vector, followed by blue-white screening. Then PCR identification was completed to select positive clones. The positively cloning bacterial suspension was sequenced by Beijing SunBio Company (Beijing, China).

Based on the sequencing results of the differentially expressed genes, the homology of nucleotide sequences and protein sequences was compared to the library of legumes using EST, BLASTN and BLASTX in the NCBI GenBank ${ }^{\circledR}$ (http://www.ncbi.nlm.nih.gov/BLAST/ Blast.cgi?CMD=Web\&PAGETYPE=BLASTHome).

\section{RESULTS AND ANALYSIS}

\section{Recycling and reverse Northern verification of differential genes}

In this experiment, differentially expressed genes were recovered from the leaves of the hybrid Jilin 38xEXP and its parents at the seedling stage using differential display technology of mRNA. To reduce the number of false positives, each primer combination was amplified twice using PCR, and only the fragments that exhibited consistent expression in both PCR amplification 
reactions and that had differential bands between hybrids and parents were recovered. A total of 120 fragments were recovered, and reverse Northern verification (Figure 1 and Table 1) showed that 22 fragments were differentially expressed between the hybrids and parents. We found that the most qualitatively differential genes in the DDRT test were quantitatively differential, as shown by reverse Northern verification. Hybridization showed that 6 of the 22 fragments had low parent expression in the hybrid, accounting for $27.2 \%$ of all differential genes. Five fragments had high parent expression in the hybrid, accounting for about $22.7 \%$ of all differential genes. Four fragments had an enhanced expression in the hybrid, accounting for about $18.2 \%$ of all differential fragments. Five fragments had a decreased expression in the hybrid, accounting for about $22.7 \%$ of all differential fragments. Two fragments were consistently expressed in a single parent, accounting for about $9.1 \%$ of differential fragments.

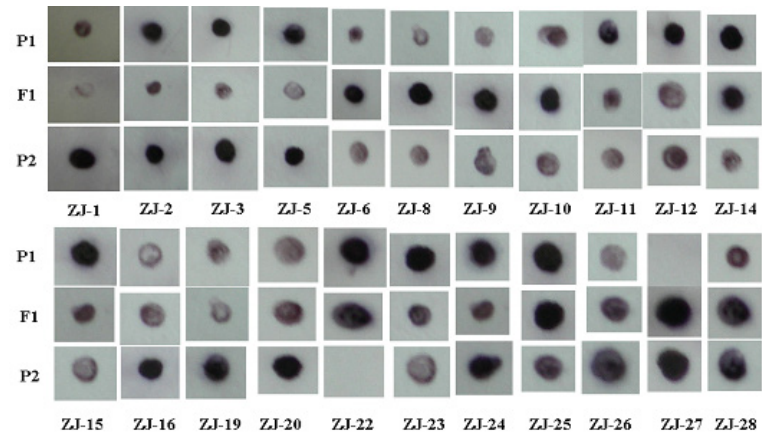

Figure 1. Reverse Northern blot hybrid of differential expression gene. P1 = female parent Jilin 38, F1 = hybrid combinations Jilin 38xEXP, P2 = male parent EXP. Combination of letters and numbers denote cloning number under diagram.

Table 1. Expression patterns of differential genes and reverse Northern hybrid.

\begin{tabular}{lcl}
\hline No. of clone & DDRT pattern & Pattern of reverse Northern hybrid \\
\hline ZJ-1 & 101 & Decreased expression of hybrid \\
ZJ-2 & 101 & Decreased expression of hybrid \\
ZJ-3 & 101 & Decreased expression of hybrid \\
ZJ-5 & 101 & Decreased expression of hybrid \\
ZJ-6 & 010 & Enhanced expression of hybrid \\
ZJ-8 & 010 & Enhanced expression of hybrid \\
ZJ-9 & 010 & Enhanced expression of hybrid \\
ZJ-10 & 010 & Enhanced expression of hybrid \\
ZJ-11 & 100 & Low parent expression of hybrid \\
ZJ-12 & 100 & Low parent expression of hybrid \\
ZJ-14 & 100 & High parent expression of hybrid \\
ZJ-15 & 100 & Low parent expression of hybrid \\
ZJ-16 & 001 & Low parent expression of hybrid \\
ZJ-19 & 001 & Low parent expression of hybrid \\
ZJ-20 & 001 & Low parent expression of hybrid \\
ZJ-22 & 110 & Consistent expression in single parent \\
ZJ-23 & 110 & High parent expression of hybrid \\
ZJ-24 & 110 & Decreased expression of hybrid \\
ZJ-25 & 110 & High parent expression of hybrid \\
ZJ-26 & 011 & High parent expression of hybrid \\
ZJ-27 & 011 & Consistent expression in single parent \\
ZJ-28 & 011 & High parent expression of hybrid \\
\hline
\end{tabular}

$110=$ Bands present in the female parent and the F1 but absent in the male parent; $011=$ bands present in the male parent and the $\mathrm{F}_{1}$ but absent in the female parent; 101 = bands present in both parents but absent in the $\mathrm{F}_{1} ; 100=$ bands only present in the female parent; $001=$ bands only present in the male parent; $010=$ bands only present in the $\mathrm{F}_{1}$. 


\section{Comparison of the homology of differentially expressed genes}

First, comparison of the sequencing results of 22 differentially expressed genes with the EST database showed that, in addition to ZJ-27, the other fragments were all highly homologous with the soybean EST (list is omitted). Then, comparison of the sequenced cloning fragment sequence with the nucleotide sequence (Table 2) and protein sequence (Table 3 ) in the GenBank showed that ZJ-3, ZJ-19, ZJ-22, ZJ-27, and ZJ-28 did not have homologous nucleotide sequences.

\begin{tabular}{|c|c|c|c|}
\hline No. of clone & Homologous analysis of nucleotide & GenBank accession GI & Identities \\
\hline $\mathrm{ZJ}-1$ & Soybean satellite DNA & GI:402616 & $85 / 93(91 \%)$ \\
\hline $\mathrm{ZJ}-2$ & $26 \mathrm{~S}$ ribosomal RNA gene of cascara etc. & GI:19919543 & $263 / 271(97 \%)$ \\
\hline $\mathrm{ZJ}-3$ & None & & \\
\hline ZJ-5 & Cloning mth2-11d24 of Medicago truncatula & GI:92874198 & $65 / 67(97 \%)$ \\
\hline ZJ-6 & Soybean root nodule acid phosphatase mRNA & GI:3341442 & $295 / 326(90 \%)$ \\
\hline ZJ-8 & Soybean chlorophyll a/b binding protein II (Cab-6) gene & GI:16805331 & $362 / 374(96 \%)$ \\
\hline ZJ-9 & $26 \mathrm{~S}$ ribosomal RNA gene of Androstachys johnsonii etc. & GI:19919564 & $264 / 271(97 \%)$ \\
\hline $\mathrm{ZJ}-10$ & Soybean chlorophyll a/b binding protein II (Cab-6) gene & GI:16805331 & $511 / 527(96 \%)$ \\
\hline $\mathrm{ZJ}-11$ & Precursor of nucleus-encoded chloroplast protein mRNA in pea & GI:169127 & $253 / 277(91 \%)$ \\
\hline $\mathrm{ZJ}-12$ & Cloning mth2-44011 of Medicago truncatula chromosome 2 & GI:47679109 & $141 / 171(82 \%)$ \\
\hline $\mathrm{ZJ}-14$ & $18 \mathrm{~S}$ ribosomal RNA gene of Galearia filiformis etc. & GI:119368163 & $147 / 149(98 \%)$ \\
\hline $\mathrm{ZJ}-15$ & Cloning mth2-7h6 of Medicago truncatula & GI:109689796 & $299 / 313(95 \%)$ \\
\hline ZJ-16 & Soybean O-acetyl-serine-(thiol)-lyase (OASTL) gene & GI: 146148621 & $156 / 159(98 \%)$ \\
\hline ZJ-19 & None & & \\
\hline ZJ-20 & Cloning mth2-7k4 of Medicago truncatula & GI:89337310 & $253 / 297(85 \%)$ \\
\hline $\mathrm{ZJ}-22$ & None & & \\
\hline $\mathrm{ZJ}-23$ & Cultivated soybean chloroplast PI 437654 gene & GI:83595723 & $241 / 247(97 \%)$ \\
\hline ZJ-24 & Cloning mth2-32j21 of Medicago truncatula & GI:61675738 & $109 / 130(83 \%)$ \\
\hline $\mathrm{ZJ}-25$ & $18 \mathrm{~S}$ ribosomal RNA gene of Malpighiaceae etc. & GI:119368160 & $207 / 210(98 \%)$ \\
\hline $\mathrm{ZJ}-26$ & Lotus corniculatus chromosome 4 clone: LjT10G08 and TM0175 & GI:29122737 & $259 / 278(93 \%)$ \\
\hline $\mathrm{ZJ}-27$ & None & & \\
\hline $\mathrm{ZJ}-28$ & None & & \\
\hline
\end{tabular}

Comparison of the ZJ-1 nucleotide sequence showed that it was homologous with soybean satellite DNA (91\%). Comparison of its protein showed that it was homologous with a hypothetical protein of Trypanosoma $(32 \%)$ and chromosome associated factors in human protein MBD1 (29\%). Therefore, ZJ-1 was assumed to be associated with soybean satellite DNA. The ZJ-2 and ZJ-9 nucleotide sequence was highly homologous with the 26S ribosomal RNA gene (97\%). Its protein sequence was homologous with the hypothetical protein of Arabidopsis (86$88 \%$ ); hence, it was inferred to be the $26 \mathrm{~S}$ ribosomal RNA gene. The ZJ-3 nucleotide sequence did not have a homologous nucleotide sequence, but its protein sequence was homologous with the AMP dependent synthesis ligase (68\%) in Medicago truncatula, and long-chain fatty acyl-coenzyme A synthetase 6 (66\%) in Arabidopsis. The ZJ-5 nucleotide sequence was highly homologous with the clonal fragment mth2-11d24 of M. truncatula (97\%), and its protein sequence was homologous with the Nmr solution structure of the partially disordered protein At2g23090 in M. truncatula (84\%), and an unknown protein in Arabidopsis (76\%).

The ZJ-6 nucleotide sequence was highly homologous with the soybean root nodule acid phosphatase mRNA (90\%), and its protein sequence was highly homologous with the soybean acid phosphatase ( $85 \%)$; therefore, it was inferred to be the soybean acid phospha- 
tase. The ZJ-8 and ZJ-10 nucleotide sequences were highly homologous with the soybean chlorophyll a/b binding protein II (Cab-6) gene (96\%), and their protein sequences were highly homologous with the soybean chlorophyll a/b binding protein II (99\%); therefore, they were inferred to be the soybean chlorophyll a/b binding protein II. The ZJ-11 nucleotide sequence was the precursor of the nucleus-encoded chloroplast protein mRNA in the pea (91\%), and its protein sequence was homologous with the hypothetical protein OsJ_014066 of Japanese rice $(97 \%)$.

\begin{tabular}{|c|c|c|c|}
\hline No. of clone & Homologous analysis of protein & GenBank accession GI & Identities \\
\hline \multirow[t]{2}{*}{ ZJ-1 } & Chromosome associated factors in human protein MBD1 & GI:119925690 & $16 / 55(29 \%)$ \\
\hline & A hypothetical protein of Trypanosoma & GI:71655753 & $21 / 65(32 \%)$ \\
\hline \multirow[t]{2}{*}{$\mathrm{ZJ}-2$} & Hypothetical protein of Arabidopsis & GI:110740129 & 77/89 (86\%) \\
\hline & Homologous senescence-associated protein in pea & GI:13359451 & $32 / 44(72 \%)$ \\
\hline \multirow[t]{2}{*}{ ZJ-3 } & AMP dependent synthesis ligase in Medicago truncatula & GI:144923456 & $26 / 38(68 \%)$ \\
\hline & Long-chain fatty acyl-coenzyme A synthetase in Arabidopsis & GI:20805873 & $22 / 33(66 \%)$ \\
\hline \multirow[t]{2}{*}{$\mathrm{ZJ}-5$} & $\begin{array}{l}\text { Nmr solution structure of the partially disordered protein At2g23090 } \\
\text { in Medicago truncatula }\end{array}$ & GI:92874232 & $33 / 39(84 \%)$ \\
\hline & Unknown protein in Arabidopsis thaliana & GI:18400164 & $30 / 39(76 \%)$ \\
\hline ZJ-6 & Soybean acid phosphatase & GI:3341443 & $88 / 103(85 \%)$ \\
\hline $\mathrm{ZJ}-8$ & Soybean chlorophyll $\mathrm{a} / \mathrm{b}$ binding protein II & GI:16805332 & $124 / 125(99 \%)$ \\
\hline ZJ-9 & Hypothetical protein of Arabidopsis & GI:110740129 & $79 / 89(88 \%)$ \\
\hline ZJ-10 & Soybean chlorophyll a/b binding protein II & GI:16805332 & $175 / 176(99 \%)$ \\
\hline ZJ-11 & Hypothetical protein OsJ_014066 of Japanese rice & GI:125590233 & $92 / 94(97 \%)$ \\
\hline $\mathrm{ZJ}-12$ & IQ calmodulin binding protein in Medicago truncatula & GI:124359355 & $73 / 102(71 \%)$ \\
\hline ZJ-14 & Unnamed protein products of Kluyveromyces & GI:50307713 & $29 / 33(87 \%)$ \\
\hline $\mathrm{ZJ}-15$ & Hypothetical protein in Magnetospirillum gryphiswaldense & GI:78033431 & $15 / 22(68 \%)$ \\
\hline ZJ-16 & Soybean PPR protein & GI:83853824 & $29 / 31(93 \%)$ \\
\hline \multirow[t]{2}{*}{ ZJ-19 } & Hypothetical protein in Arabidopsis or PPR protein $(65 \%)$ & GI:21780142 & $83 / 126(65 \%)$ \\
\hline & & GI: 15227067 & $83 / 126(65 \%)$ \\
\hline \multirow[t]{2}{*}{$\mathrm{ZJ}-20$} & Similar subtilisin in peanut; Protease-associated PA in Medicago truncatula; & GI:66735598 & $82 / 96(85 \%)$ \\
\hline & protease inhibitor $\mathrm{I} 9$, subtilisin precursor & GI:140067279 & $80 / 96(83 \%)$ \\
\hline $\mathrm{ZJ}-22$ & A hypothetical protein OsJ_027731 in Japanese rice (55\%), GI:125605212 & GI:115478555 & $110 / 197(55 \%)$ \\
\hline $\mathrm{ZJ}-23$ & Soybean photosystem I P700 apoprotein A2 & GI:91214134 & $66 / 68(97 \%)$ \\
\hline $\mathrm{ZJ}-24$ & IQ calmodulin binding protein CG-1 in Medicago truncatula & GI:144923073 & $69 / 91(75 \%)$ \\
\hline \multirow{2}{*}{$\mathrm{ZJ}-25$} & CHK1 checkpoint homolog (S. pombe) & GI:89271365 & $30 / 34(88 \%)$ \\
\hline & TO71-3 sequence in dandelion & GI:75755995 & $30 / 34(88 \%)$ \\
\hline \multirow[t]{2}{*}{$\mathrm{ZJ}-26$} & A hypothetical protein OsJ_014066 in Japanese rice & GI:125590233 & $92 / 95(96 \%)$ \\
\hline & Chloroplast proteolytic enzyme ClpA (CD4B) & GI:399213 & $93 / 97(95 \%)$ \\
\hline $\mathrm{ZJ}-27$ & SJCHGC09122 protein of Schistosoma japonicum & GI:76155528 & $16 / 53(30 \%)$ \\
\hline $\mathrm{ZJ}-28$ & Aldehyde dehydrogenase in Medicago truncatula & GI:92891585 & $136 / 172(79 \%)$ \\
\hline
\end{tabular}

The ZJ-12, ZJ-15, ZJ-20, and ZJ-24 nucleotide sequences were homologous with the clonal fragment of $M$. truncatula. The ZJ-12 and ZJ-24 protein sequences were homologous with the IQ calmodulin binding protein in M. truncatula. The ZJ-15 sequence was homologous with the hypothetical protein in Magnetospirillum gryphiswaldense (68\%). The ZJ-20 sequence was homologous with a similar subtilisin in the peanut (85\%). The ZJ-14 and ZJ-25 nucleotide sequences were highly homologous with the $18 \mathrm{~S}$ ribosomal RNA gene (98\%). The ZJ-14 protein sequence was homologous with an unnamed protein product of Kluyveromyces $(87 \%)$. The ZJ-25 protein sequence was homologous with the CHK1 checkpoint homo$\log$ (Schizosaccharomyces pombe) and the TO71-3 sequence in dandelion (88\%). The ZJ-16 nucleotide sequence was highly homologous with the soybean O-acetyl-serine-(thiol)-lyase gene $(98 \%)$, and its protein sequence was highly homologous with the soybean PPR protein (93\%). The ZJ-19 and ZJ-22 nucleotide sequences did not have homologous sequences, but 
their proteins were homologous with a hypothetical protein in some plants.

The ZJ-23 nucleotide sequence was highly homologous with the cultivated soybean chloroplast PI 437654 gene (97\%), and its protein sequence was highly homologous with the soybean photosystem I P700 apoprotein A2 (97\%); hence, it was inferred to be a regulatory protein in soybean photosynthesis. The ZJ-26 nucleotide sequence was highly homologous with the Lotus corniculatus chromosome 4 clone (LjT10G08 and TM0175) (93\%), and its protein sequence was highly homologous with a hypothetical protein OsJ_014066 in Japanese rice (95\%) and chloroplast proteolytic enzyme ClpA (CD4B) (95\%). The ZJ-27 sequence was not homologous with any sequences in the EST library or the nucleotide library; however, its protein sequence was homologous with the SJCHGC09122 protein of Schistosoma japonicum, to a certain extent (30\%). The ZJ-28 sequence did not have any homologous nucleotides; however, its protein sequence was highly homologous with aldehyde dehydrogenase in $M$. truncatula $(79 \%)$.

\section{DISCUSSION}

The differential expression pattern of cDNA fragments may be divided into enhanced expression in the hybrid, decreased expression in the hybrid, high parent expression, low parent expression, and consistent expression in a single parent. The relationships among these different groups are considered here.

In the enhanced expression of genes derived from reverse Northern hybrid expression patterns, the DDRT expression pattern is the specific hybrid model ( 010 , see notes in Table 1$)$. We completely analyzed 4 fragments, namely, ZJ-6, ZJ-8, ZJ-9, and ZJ-10, which were found to be soybean acid phosphatase, 2 soybean chlorophyll a/b binding protein II, and a $26 \mathrm{~S}$ ribosomal RNA gene, respectively. These genes had particularly obvious heterosis yields, which might contribute toward improving soybean yield for specific hybrid fragments. Li and Lv (2005) showed that the soybean acid phosphatase (APA) is one of the important enzymes in plants, and that it plays an important role in the conversion of carbohydrate and the synthesis of protein. In addition, this enzyme is closely related to the decomposition and utilization of soil organic phosphorus and the reutilization of $\mathrm{P}$ in the plant.

Liu et al. (1999) found that when phosphorus (P) is limited, the APA activity in plants is exponentially increased. Different varieties of different crops might use soil P efficiently through different physiological mechanisms. Therefore, the APA in soybean might indirectly promote soybean yield by improving $\mathrm{P}$ utilization. The chlorophyll $\mathrm{a} / \mathrm{b}$ binding protein in soybean is involved in the acquisition and transmission of light energy (Sun et al., 2000). This protein allows soybean to enhance its absorption and transmission efficiency of light energy (Liu et al., 2004), and then provides the material and energy required to improve yield. It is well known that ribosome is an important organelle for protein synthesis. Therefore, $26 \mathrm{~S}$ might be involved in the process of protein synthesis, and might also play a role in the positive regulation of yield, to a certain extent.

In the decreased expression of genes through the reverse Northern hybrid expression patterns, most DDRT expression patterns are expressed silently in both parents (101, see notes of Table 1). We cloned the fragments that had decreased expression in the hybrid (ZJ-1, ZJ-2, ZJ-3, ZJ-5, and ZJ-24) and, through the comparison of their homology, found that some were highly homologous with the hypothetical protein of Arabidopsis, some were highly homolo- 
gous with the AMP dependent synthesis ligase in M. truncatula, and some were homologous with the inferred senescence-associated protein in the pea and chromosome-associated factors in the MBD1 of the human protein. These regulatory proteins or enzymes have an important effect on the structure, function, and evolution of soybean genome.

In the studies focusing on differential gene expression between interspecific hybrids and their parents, Kashkush et al. (2002) found 2 retrotransposons in the hybrids; however, they were differentially expressed in the genes of hybrids. This difference might be influenced by high genetic differences between different species. As a result, interspecific hybrids must coordinate gene expression between different genomes by activating some new genes, to overcome various adverse effects, such as reduced fertility. The differentially expressed genes of chromosome associated factors in the MBD1 of the human protein that were cloned in the current experiment with decreased expression in the hybrid might be transcriptional repressors (Ichimura et al., 2005) that ultimately coordinate the whole growth process.

Four fragments cloned in this experiment had high parent expression in the hybrid, namely, ZJ-23, ZJ-25, ZJ-26, and ZJ-28. Two genes had consistent expression in the parents and hybrids, namely, ZJ-22 and ZJ-27. These 2 genes had DDRT expression patterns that were consistent in a single parent (110 or 011, see notes of Table 1). ZJ-22 and ZJ-27 did not have a homologous nucleotide, while the protein products had an unknown effect. ZJ-23 is the soybean photosystem I P700 apoprotein A2, which might regulate photosynthesis by regulating the reaction center cytochrome P700 of the photosynthetic system I (Khan et al., 2007). ZJ-26 is a hypothetical protein in Japanese rice, which is highly homologous with the chloroplast proteolytic enzyme ClpA (CD4B). As an ATP dependent proteolytic enzyme, C1p plays an important role in the metabolic control of bioproteins, controls the metabolism process by regulating the rate-limiting enzyme level in the metabolic pathway, and also promptly removes some intracellular proteins that have potential toxicity or cause irreversible damage, to ensure the normal physiological functions of cells. In addition, multiple subunits of chloroplast proteases have been shown to be involved in the senescence process; however, the mechanism of chloroplast protease on senescence has yet to be elucidated (Wang and Sun, 2005). ZJ28 is highly homologous with the aldehyde dehydrogenase in M. truncatula, which may be related to salt tolerance in soybean. Cui and Diao (2005) cloned the betaine dehydrogenase gene in spinach, and confirmed that an increase in betaine is related to betaine aldehyde dehydrogenase (BADH) activity. In addition, the BADH gene enhances the salt tolerance of plants.

Six fragments were cloned in this study that had low parent expression, namely, ZJ11, ZJ-15, ZJ-16, ZJ-19, and ZJ-20. The DDRT patterns of these fragments were silently expression in a single parent (100 or 001). The functional fragments included the hypothetical protein in Arabidopsis, Kluyveromyces, M. gryphiswaldense, and rice, in addition to the IQ calmodulin binding protein (CaMBP) in M. truncatula and the soybean protein PPR. The PPR (pentatricopeptide repeats) gene family is one of the largest families found in plants. PPR has 35 amino acids that are continuously arranged together as a repeating unit. This gene might contribute to mitochondrial or chloroplast regulation in combination with the organelle transcript (Xu et al., 2006). As the most important $\mathrm{Ca}^{2+}$ sensor, CaMBP regulates the physiological functions of cells by affecting its downstream CaMBP (Mao et al., 2004). Some studies have shown that the calmodulin gene might be involved in salt induction (Huang et al., 2004). The effect of the other hypothetical proteins and regulatory factors requires further study. 


\section{REFERENCES}

Burns WC, Maitra N and Cushman JC (1996). Isolation and characterization of a cDNA encoding a LEA5-Like protein from soybean (U66316). Plant Physiol. 112: 1398.

Cerna FJ, Cianzio SR, Rafalski A, Tingey S, et al. (1997). Relationship between seed yield heterosis and molecular marker heterozygosity in soybean. Theor. Appl. Genet. 95: 460-467.

Cheng NH, Hu JG and Zhao XS (1997). Gene express patterns of light-grown and dark-grown seedlings maize and soybean. J. Fudan Univ. (Nat. Sci.) 36: 524-530.

Cui RL and Diao XM (2005). Advances on cloning and translation of the salt tolerance genes in plant. China Biotechnol. 25: $25-30$.

Huang J, Mullapudi N, Sicheritz-Ponten T and Kissinger JC (2004). A first glimpse into the pattern and scale of gene transfer in Apicomplexa. Int. J. Parasitol. 34: 265-274.

Ichimura T, Watanabe S, Sakamoto Y, Aoto T, et al. (2005). Transcriptional repression and heterochromatin formation by MBD1 and MCAF/AM family proteins. J. Biol. Chem. 280: 13928-13935.

Kashkush K, Feldman M and Levy AA (2002). Gene loss, silencing and activation in a newly synthesized wheat allotetraploid. Genetics 160: 1651-1659.

Khan H, Kozera C, Curtis BA, Bussey JT, et al. (2007). Retrotransposons and tandem repeat sequences in the nuclear genomes of cryptomonad algae. J. Mol. Evol. 64: 223-236.

Li CB and Lv SF (2005). Research summarization on acid phosphatase in higher plants. J. Xinyang Agr. Coll. 15: 88-89.

Li JX, Yu SB, Xu CG and Tan YF (2000). Quantitative trait loci (QTLs) analyses of yield and its component traits in "Sanyou63" hybrid. Acta Agron. Sincia 26: 892-898.

Liang P and Pardee AB (1992). Differential display of eukaryotic messenger RNA by means of the polymerase chain reaction. Science 257: 967-971.

Liu H, Liu JF and Liu WD (1999). Differences of root morphology and physiological characteristics between two rape genotypes with defferent p efficiency. Plant Nutr. Fert. Sci. 5: 40-45.

Liu Z, Yan H, Wang K, Kuang T, et al. (2004). Crystal structure of spinach major light-harvesting complex at 2.72 A resolution. Nature 428: 287-292.

Mao GH, Song LX and Sun DY (2004). Progress of study on calmodulin-binding proteins in plants. Zhi. Wu Sheng Li Yu Fen. Zi. Sheng Wu Xue. Xue. Bao. 30: 481-488.

Ni ZF, Sun QX and Wu LM (2000). Differential gene expression between wheat hybrids and their parental inbreds in seedling leaves of early and vigorous tillering stages. J. China Agr. Univ. 5: 1-8.

Schopfer CR and Ebel J (1998). Identification of elicitor-induced cytochrome P450s of soybean (Glycine max L.) using differential display of mRNA. Mol. Gen. Genet. 258: 315-322.

Stuber CW (1995). Mapping and manipulating quantitative traits in maize. Trends Genet. 11: 477-481.

Sun QC, Leng J and Li LB (2000). Recent advances of studies on the structure and function of the light-harvesting chlorophyll a/b-protein complex. Chin. Bull. Bot. 17: 280-301.

Vladimir E (2001). Genes Differentially Expressed in Soybean Lines Sensitive and Tolerant to Aluminium Stress. PhD Dissertation of Institute of Plant Genetics and Crop Plants Research. Gatersleben.

Wang ZH and Sun YQ (2005). Progress in study on clp protease. Pharm. Biotechnol. 12: 412-415.

Wang YL, Xi GS and Wang PW (2008). Heterosis ana lysis by using different soybean as parents. Soybean Sci. 27: 760763.

Wu LM (2004). Thesis: Relationship Between Differential Gene Expression Patterns and Heterosis in a Wheat Diallel Crosses. Chinese Agricultural University, Beijing.

Xiao J, Li J, Yuan L and Tanksley SD (1995). Dominance is the major genetic basis of heterosis in rice as revealed by QTL analysis using molecular markers. Genetics 140: 745-754.

Xiong LZ (1999). Studies on Molecular Basis of Rice Heterosis at Gene Expression Level. PhD Dissertation of Huazhong Agricultural University. Huazhong Agricultural University, Wuhan.

Xu XB, Qiu DL, Sun YT, Wang SJ, et al. (2006). Advance in the study of PPR gene family. Yi. Chuan 28: 726-730.

Zhang B, Qiu LJ and Chang RZ (2003). Primary study on predicting heterosis by SSR marker distance among soybean cultivars. Soybean Sci. 22: 166-171.

Zhang J, Wang PW, Yan DS, Yang XB, et al. (2010). Relationship between differential gene expression patterns and heterosis in soybean seedling stage. J. Northwest A\&F Univ. (Nat. Sci.) 38: 91-100.

Zhao M, Sun Z, Wang SM, Wang YQ, et al. (2004). Breeding of hybrid soybean HybSoy 1. Chin. J. Oil Crop Sci. 26: 15-17. 